\title{
An ontology for analysing cognition in geometric and parametric design platforms: a review
}

\author{
Rongrong $\mathrm{YU}^{1}$, Oluwole Alfred OLATUNJI* (PhD, MRICS, RQS) ${ }^{2}$, Abiola \\ Abosede, AKANMU (PhD, $)^{3}$
}

${ }^{1} \mathrm{PhD}$ Candidate, Department of Architecture, University of Newcastle, Callaghan, 2308, NSW Australia, Email: Rongrong.yu@uon.edu.au

${ }^{2}$ Lecturer, Department of Construction Management, Curtin University, GPO Box U1987, Perth, WA 6845, Australia, PH (61) 9266-7492, Email: oluwole.olatunji@curtin.edu.au

${ }^{3}$ Assistant Professor, Department of Civil and Construction Engineering, Western Michigan University, 1903 W. Michigan Ave., Kalamazoo, MI 49008-5316, Phone: (269) 276-3201, Email: abiola.akanmu@wmich.edu,

* Correspondence author

\begin{abstract}
Things are changing: designers are now drawn between geometric modelling environment (GME) and the parametric design environment (PDE). Neither of these is a replacement or a substitute to the other. Moreover, non-design disciplines are also responding to this change: construction managers and estimators are now required to have the ability to interact with CAD drawings and design models. This has become necessary so that they can distil the robustness of digital design platforms as they ingrain the compatibility and accuracy of their deliverables (e.g. object-based estimating, virtual reality and process simulation). In essence, new theories on digital processes and methods are being primed on the management of data in digital platforms rather than the iteration of conventions as in manual processes. The aim of this study therefore is to add to the evolving knowledge on this subject by evocating an ontology for analysing cognitive processes in geometric modelling and parametric project environments. The significance of this is critical: it adds value on the framework for multidisciplinary interaction within a BIM project team; towards a synchronous cognition processes within design and non-design disciplines. It also adds depth to extant methodological debates in multidisciplinary research studies. Further areas of research are elicited in the conclusion of the study.
\end{abstract}

Keywords: computer-aided design, digital estimating methods, protocol analysis, geometric modelling environment, parametric design environment.

\section{INTRODUCTION}

Modern project development culture is now primed on the integration and collaboration within project teams. The integration requires non-design disciplines to be closer to design cognition processes, especially as design platforms continued to change consistently. Olatunji (2012) has elicited this in a clearer context: non-design disciplines should be able to access design tools with the intention to restructure 
design models in ways that appropriately represent projects' best value systems. These have become expedient as design models are not structured to meet specific discipline's requirements, rather to represent the physicality of a finished product. Moreover, both the design and the non-design disciplines have always had different roles to play in project development processes. Regardless, digital design tools are diminishing the boundary between both disciplinary domains as though both domains have had to contribute to the integrity of the design model through the principle of multidisciplinary integration, rather than through the conventional fragmented culture. On the other hand, project success in parametric design thrives on collaboration, and partners cannot collaborate unless there is some form of cross-disciplinary integration or thorough understanding of other team-partners' cognition processes. Meanwhile, there are some challenges are relative to this, including:

- Collaboration is not a hallmark of the construction industry (Ashcraft, 2008). By default, professional disciplines in the construction industry are independent entities, and have had considerable commitment to fragmented processes and systems. This has often resulted in having to struggle with the ethos of collaboration. The challenge has also triggered several constraints to project performance; including value sharing and communication issues, dysfunctional relationships and the susceptibility of projects to failure (Pinto and Mantel, 1990).

- The gap created by fragmented conventions in the industry is also markedly challenging; construction professional service processes have had to be reengineered, but gradually so e.g. by reskilling, continuing support and through a soft realignment of business systems (Olatunji, 2011)

This research aims to contribute to existing scholarly debates regarding multidisciplinary collaboration in the construction industry. In particular, it focuses on comparing and interpreting designers' cognitive processes in geometric modelling and parametric design environments in such a way that professionals from non-design disciplines are able to understand these cognition processes. Once the cognition gap is bridged, integration and collaboration will become easier.

\section{Designer's and non-designer's cognition processes}

Traditionally, the integrity of construction management processes is primed on the ability of practitioners within the non-design domain to accurately interpret and implement design - its principles, intentions, contexts, conventions and attributes. Interpreting design intentions is a momentous challenge. Professionals who are not designers are simply not designers; they often can only imagine designers' actual intentions, and the precision of such imaginations is usually a critical issue (Ashworth, 2010). Even where there is an integration of the design and design management processes, the gap in the cognition processes of both disciplines is evidently significant. For example, the many design disciplines involved in project development processes think differently when creating design solutions, and they use different tools that are usually not interoperable (Jadid and Idrees, 2007; Kwon et al., 2009). A key implication of this is that there is a wide margin of variability: (1) for non-designers to master the rapidly changing conventions in the many design applications; and (2) for non-designers to adapt the rapid changes within their own disciplines, to the variability in the design disciplines. This challenge is huge: it involves using many software applications, usually fragmented, requiring considerable cognitive effort in distilling the different design intentions and principles 
into context-specific scenarios of implementation (Dean and McClendon, 2007).

The gap between the respective disciplines' cognition processes becomes significant, especially considering the processes involved in creating extensively innovative solutions for projects. Emerging design tools, parametric tools in particular, allow designers to create complex design solutions with extreme flexibility and efficiency. These are not simply the traditional CAD approaches to designing: unlike traditional $\mathrm{CAD}$, generally, parametric tools are rule-based and are flexible for change. They also offer parallel developing solutions, just as they promote efficiency and are specialized on free-form generation (Woodbury, 2010). Consequently, and arguably so too, parametric tools have triggered the widening of the gap between designers cognition processes in the traditional design contexts and the world of the modern designer. The various arguments on the efficacy of parametric design processes are reviewed below. The review provides a lead on the theoretical framework for analysing designers' cognition processes while creating extensively innovative solutions for designers and estimators within the parametric design environment (PDE).

\section{The efficacy of parametric design methods and designers' cognition processes}

Parametric design is a dynamic rule-based process, controlled by variations of parameters by which multiple design solutions can be developed concurrently. The term "parameters" means factors which determine a series of variations in design forms. In architecture, such are usually defined as building parameters or environmental factors. Some CAD application may have varying parametric components as tools and / or attributes; however they are not entirely classified as parametric applications. This is because their design development processes are fragmented, and they are not made to conform to parametric rule algorithms (Whyte et al., 2000; Yang and Han, 2006). According to Woodbury (2010), parametric supports the creation, management and organization of complex design models. Kolarevic (2003) have previewed what this potentiality is: while using parametric design tools, designers can make rules according to the performance requirements of a design. When the parameters underlying an object are changed, particular instances can be altered or created from a potentially infinite range of possibilities. Such deliverables can be expressed in terms of the ability to simplify very complex communications.

Parametric design is not entirely the same from conventional design methods both in terms of design processes and outcomes. Although both of them serve different purposes, as shown in Figure 1, parametric design tools come with several attributes that facilitate the simplification of complex solution in ways that improve efficiency, productivity, quality of outcome and creativity. Many thought-provoking questions have been asked recently by stakeholders in the design industry exploring the difference between conventional design methods and parametric concepts. The overarching conclusion from such debates is as though either of the two is neither a substitute nor a replacement for the other (Zyskowski, 2009). Moreover, another vital interest of this community is to determine whether or not parametricism - i.e. exploring parametric design principles by way of concepts, methods and environment - does enhance or hinder designers in their design processes. To understand the merits of a design tool, an effective method is by studying designers' cognitive behaviour when they use the tools (Gero and Mc Neill, 1998). 


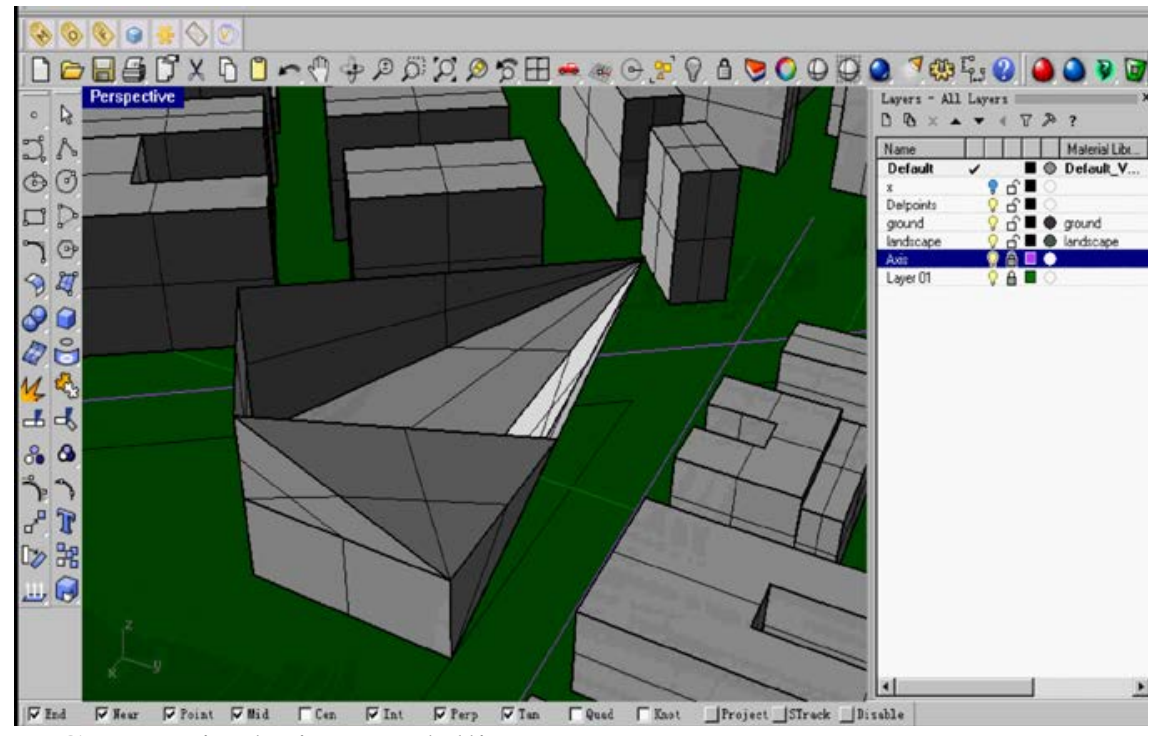

Geometrical modelling has no scripting interface; all data are built directly into the model. As a result, there is a limit to the absorptive capacity of the platform, and designers struggle with seamless expression of complex communications.

a: Geometric design modelling

Most software applications used in parametric design platforms do have the scripting interface which primes the structuring of the geometric interface. In essence, the scripting interface is built to interlink design parameters, and handle (e.g. mapping) complex data management processes. They also actuate and automate design solutions - these are nearly impossible is geometric modelling environment.

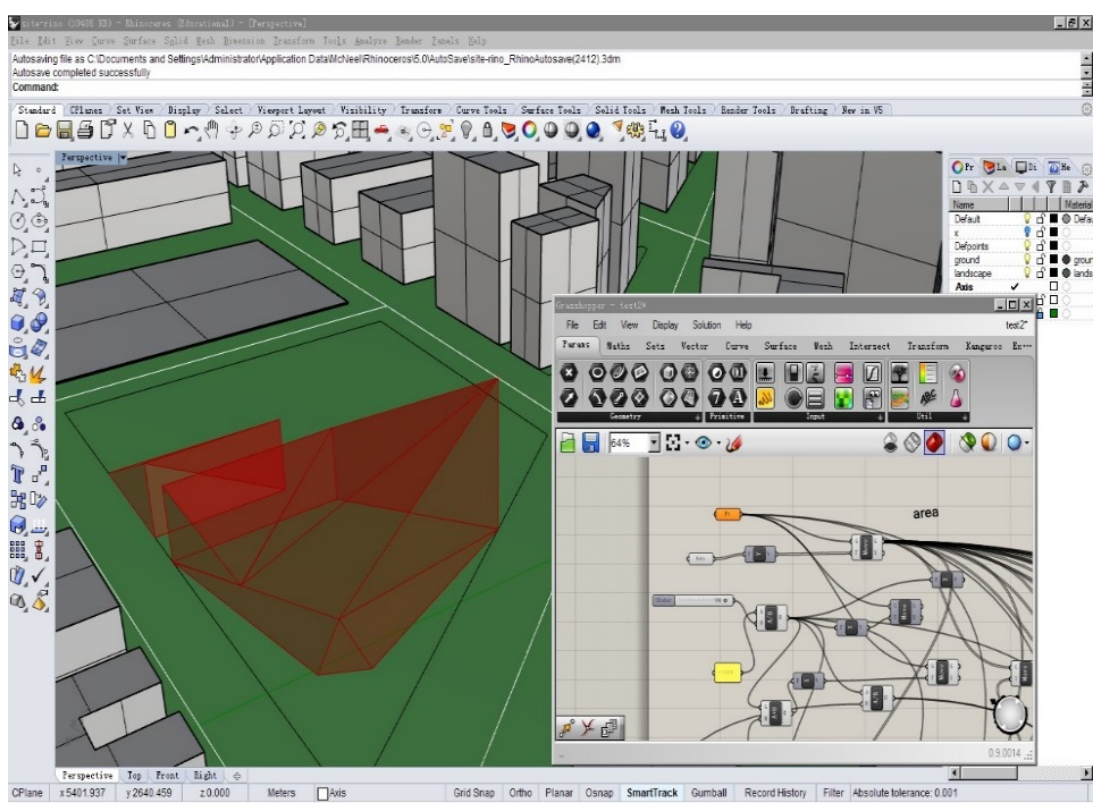

b: Parametric design modelling

Figure 1: Distinguishing geometric design modelling and parametric design modelling

Previous studies on designers' cognition process in parametric design environments (PDEs) suggest that parametric tools advance design in a variety of ways. For instance, there is evidence that the generation of ideas is positively influenced in a PDE. In particular, Iordanova et al.'s (2009) experiment on generative methods shows that ideas were shown to be generated rapidly while they also emerge simultaneously as variations in PDEs. Schnabel (2007) also showed that PDEs are beneficial for generating unpredicted events and for accommodating changes. In addition to these, Lee et al (2012) have demonstrated the use of protocol analysis to evaluate creativity in the PDE, in which case the authors find a strong positive correlation between the PDE and design creativity. This same outcome was confirmed by Chien and Yeh (2012): the authors have used parametric methods to explore "unexpected outcomes" in the PDE. The results of the study clearly confirm the viability of parametric design methods, and the potentiality of PDE to enhance creativity. 
Creativity might mean different things to different people. However, the overarching influence of creativity, as in the context of PDE, is that it unsettles practice dynamics in non-design disciplines, especially where the reliance on design disciplines' concepts and methods had always been a smooth linear relationship (Maher, 2008). Whether it is by way of creating new knowledge or by way of triggering an awareness of what has changed, or is currently changing, in the design discipline, it is important to integrate this phenomenon into the cognition spaces of practitioners within the non-design disciplines. A review on this is provided below.

\section{Non-Designers' cognition processes}

Clients, and indeed all project stakeholders, often want to visualize costs. This is an emerging knowledge area which requires estimators to show cost descriptors graphically within project models. This also means non-designers are required to have a functional skill level on how to manipulate design authoring tools, and how to deal with the deliverables arising from same. On the one hand, understanding how designers think adds a lot of value to non-designers in performing their duties within PDEs. The cross-disciplinary benefits of this are vitally critical: potentially, project outcomes are strengthened as project team members share value and integrate processes (Flager et al., 2009; Gujarathi and Ma, 2011). On the other hand, a definitive understanding of non-designers' cognition processes opens up new latitude of knowledge, and this could change the entire landscape of their practice. For example, if estimators have to perform their function through parametric tools, and they rely only on PDEs for this, this in itself creates a gap between traditional estimators and the modern-day estimators within the PDE. For the later to succeed, understanding and contextualising parametric designers' cognition processes are both crucial. In the conclusion of Olatunji (2014), the author emphasized that the future of estimating practice is no longer ingrained in the mastery of traditional design conventions and symbols, rather a conscious assimilation of how to manage seamless digital data flow within multidisciplinary platforms. Meanwhile, more importantly, such principle of data management is useless if design intentions and principles are not infused into digital estimating processes (Olatunji, 2012).

Nonetheless, such opinions as above cannot overshadow the necessity to understand non-designers' cognition processes and how such reinforce or contradict parametric designers' thought processes. An important way to look at this is to consider the multi-perspective nature of estimating practice: there are different views to how estimators think, especially as fuelled by the business principles of the different parties who might show commercial or economic interests on a construction project (Olatunji, 2012). Regardless however, a common feature to all estimators is the desire to visualize project consumables and the associated risks through design representations. In a lot of cases, it has been impossible to predict the accuracy of such visualization, or the procedures leading to achieving a perfect estimate. Interestingly, only a few researchers have explored why and how estimators take certain positions to achieve their outcome and not the other. For example, Skitmore and Patchell (1990) observed that estimating is not usually a linear process because estimators have had to consider several factors outside the design descriptors to deliver a winning outcome. Meanwhile, this is not a conclusive view: Skitmore and Wilcock (1994) took a step further; they explored how small builders develop their estimates. They found that accuracy of designs is critically important, but this must 
relate to the estimators' considerations of risks and uncertainties. The cognition process captured by these authors have had its limitations: (1) the methodology they used did not address the potential variability arising from design intentions and processes, and; (2) the participants they observed have focused more on the business intricacies of estimating outcomes, which seemed to envelop their technicalities around the efficacy of project designs in stimulating the integrity of estimating outcomes.

\section{Methodology: Protocol Analysis}

Protocol analysis is a method for turning qualitative verbal and gestural utterances into analytical data (Ericsson and Simon, 1993; Gero and Mc Neill, 1998). It has been used extensively in design research to develop an understanding of design cognition (Atman et al., 1999; Kan and Gero, 2008). Why is this important to the entire project team, both the design and the non-design disciplines? There are cases in which non-designers need to make changes to authoring or manipulation tools. To achieve this, they must accurately interpret and work only with the original intentions of the designers. Modern project development processes are predicated on integration: designers and non-designers are expected to have the ability to work well in design environments.

Usually, concurrent and retrospective protocol collection methods can be applied in design experiments. A concurrent protocol involves participants in an experiment verbalising their thoughts when designing on a specific task - also called "think aloud" - whereas a retrospective protocol explores what designers were thinking while designing, applied when the design task has been completed. Some studies have compared these two. In particular, Kuusela and Pallab (2000) argue that concurrent protocols are more suitable for examining the design process and can generate more numbers of segments, while retrospective protocols are more suitable for examining design outcomes. Another typical example is Gero and Tang's (2001) study exploring design processes. Their results show that concurrent and retrospective protocols lead to very similar outcomes in terms of exploring designers' intentions during design processes. Although, observably, both methods can be driven by design processes and contents, they authors also conclude that concurrent protocols are an efficient and applicable method by which to understand design processes. Retrospect protocol is commonly believed to be less intrusive to the design processes.

Depending on research goals, most researchers would have slightly different protocol descriptors. However, such flexibility does not excuse the sufficiency required to prime valid outcomes from protocol studies. Interestingly, there is very little confusion about this in the literature on protocol studies: most protocol studies have started with a definite direction regarding the intention of the observations within the protocol experiments (Dorst and Dijkhuis, 1995; Gero and Tang, 2001). According to Akin (1986), a protocol is the record of behaviours of designers using sketches, notes, videos or audio. After collecting the protocol data, certain coding scheme will be applied to categorise the collected data, enabling detailed study of the design process in the chosen design environments. As Gero and Tang (2001) state, protocol analysis has become the prevailing experimental technique for exploring the understanding of design. 


\section{FBS ontology, a tool for coding designers' behaviour}

A protocol study involves the encoding and analysing data collected within design environment. Many coding schemes, covering several perspectives, have been nominated by some researchers as the best way to do this. One of such is the FBS ontology developed by Gero (1990). Later, Gero and Kannengiesser (2004) further refined the ontology as six variables and eight design processes (see Figure 2 which shows the FBS ontology Gero and Kannengiesser, 2004). The variables include Function $(\boldsymbol{F})$, which is defined by the context-specific purpose and the intention that the design is projected to meet. Function means the design purpose or intention. Another variable is Behaviour (B), and this represents the way to achieve the function or structure, either expected (Be) or derived (Bs) from the structure. Structure (S) represents how the components of a model object have been structured, and the relationships between them. Two more variables can be added to the original FBS ontology: Designers' consideration on the design brief provided (coded as $\boldsymbol{R}$ ), and the design documentation process (coded as $\boldsymbol{D}$ ).

Functions $(\boldsymbol{F})$ emerge from the design requirements or designers professional knowledge. The designers' behaviours (Be) is formulated from the Function (F) or Requirement $(\mathrm{R})$ to set up goals or the way to achieve those $(\boldsymbol{B} \boldsymbol{e})$. Designers review and evaluate the existing geometry model (in the case of geometric modelling environment and parametric design environment) or scripting (only in the parametric design environment) (S), and derived their analysis on these (Bs). Through the evaluation between their expectation (Be) and analysis results (Bs), the new solution would be generated. The FBS ontology is claimed to be a universal coding scheme for various design environments (Kan \& Gero, 2009). By calculating the transitions between design variables, various analyses can be conducted.

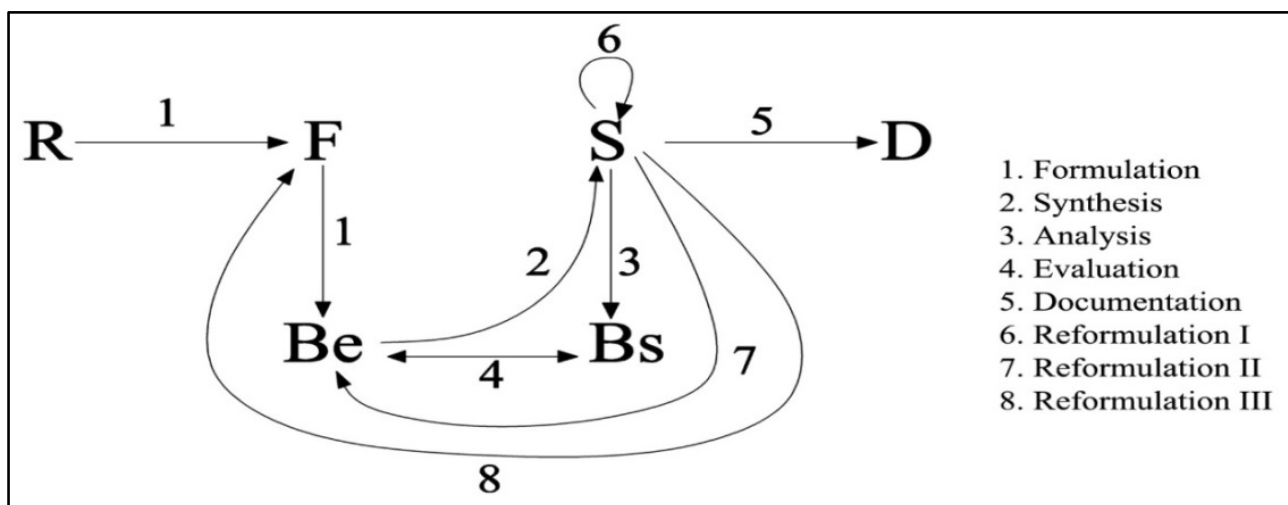

Figure 2: Ontology for protocol experiment and analysis. Adapted from (Gero and Kannengiesser, 2004)

\section{Applications}

The FBS ontology applies to designer as it does to non-designers. Both domains of cognitive space should be able to integrate knowledge and be able to collaborate effectively. Unlike in the design discipline where the margin for creativity is considerably huge, researchers on design management issues have had limited motivations to deeply and objectively explore non-designers' thought processes leading to satisfactory outcomes. It is not that such deliverables are insignificant or impossible to achieve, rather it would seem that non-designers have not been 
significantly pressured - or have not yielded significantly to the pressure - by clients requiring them to create better solutions. Such stance has received widespread criticisms - see Davis et al. (2009) and Sutrisna et al. (2005). In lieu of exploring how estimators think and the technical rationales behind the procedures for improving estimating processes, most studies have concentrated on refining certain categorical enablers of feasibility, precision and accuracy. For example, Ogunlana (1989), in a way similar to protocol analysis, has explored the factors leading to accuracy in construction estimates. The author concluded that, rather than trusting in automated processes, estimators are more inclined to relying on their previous experiences. However, technically experiences are not only difficult to acquire and transmit, there is a limit to how they help: in an industry with constantly varying circumstances, the best experience might not be very distinguishable from being a novice unless such experience is consistent with new knowledge domains and the associated circumstances that have changed or are being changed. Lowe and Skitmore (1994) have also observed that the quality of experience, in gauging estimators' thinking processes, is relative to process iterations and other factors that may be involved in improving target outcomes. Extending this view, Lowe and Skitmore (2007) observed that the thinking processes of estimators regarding the efficacy of estimating outcomes is relative to organizational culture on knowledge acquisition and diffusion. Such culture had been explained by Cohen and Levinthal (1990) to include the capacity to absorb knowledge within an organizational system as well as the capacity to generate, retain and transfer new knowledge across different organizations. When there are new knowledge and new challenges, there is bound to be new dimensions to bridging the knowledge gaps.

The importance of this is incontrovertible. Rather than pockets of conventional knowledge champions, the contemporary construction industry requires that project team partners are able to work together effectively. This is impossible unless there is openness to how knowledge is gained and used within an integrated platform. The main issue for consideration in further research is how this is to happen. The review has shown that while there are well-structured frameworks on the analysis of cognition processes within design disciplines, most design management disciplines have been vague on this. This lack of information is making it difficult for design management professionals to integrate themselves into the new world of parametricism. Going forward, we recommended that empirical research studies be dedicated to observing the reactivity of design and non-design practitioners to the FBS ontology shown in Figure 2.

\section{Conclusion}

Designers think differently; and the nuances from this even become more populated given the fact that a typical project team also involve several non-design disciplines. There is compelling evidence in recent studies suggesting that practitioners from non-design disciplines are not expected to work with design models. They now manipulate models, just as they directly interact with authoring tools. To achieve optimum integration (of practice expectations and knowledge), it is expedient that these professionals correctly understand and interpret existing theories on designers' cognitive actions. Such need becomes more critical as the innovations reposed in the nuances within designers' cognition processes are also promoted by the different tools they use. Although still being debated, as shown in the review study, 
the design discipline has had a well-structured approach to analysing cognitive processes. In the case of design management disciplines, 'experience' has been identified as the single most important factor in cognition analysis. One of the critical issues worth considering for future studies is whether the FBS ontology can be effective across multidisciplinary boundaries. Apparently, it helps that non-designers are able to use the ontology to understand how designers think and innovate, however will the variables have the same applications across the different disciplines within a project team? If they do, how will previous experiences that are not relative to FBS ontology integrate into modern design environments? If the applications are not the same across the disciplines, how can multi-disciplinary interactions within contemporary design environments be projected to a new realm of common frameworks through which collaboration and integration process could become reasonably congruent?

\section{References}

Akin, O. (1986). "Psychology of Architectural Design " Pion, London.

Ashcraft, H. W. (2008). Building Information Modeling: A Framework for Collaboration. Construction Lawyer 28, 1-14.

Ashworth, A. (2010). "Cost Studies of Building," 5th/Ed. Pearson Prentice Hall, Harlow, UK.

Atman, C. J., Chimka, J. R., Bursic, K. M., and Nachtmann, H. L. (1999). A comparison of freshman and senior engineering design processes. Design Studies 20, 131-152.

Chien, S.-F., and Yeh, Y.-T. (2012). On Creativity and Parametric Design---A preliminary study of designer's behaviour when employing parametricdesign tools. In "eCAADe2012", pp. 245-253. eCAADe in cooperation with Mediatecture, Czech Republic.

Cohen, and Levinthal (1990). Absorptive capacity: A new perspective on learning and innovation. Administrative Science Quarterly 35, 128-152.

Davis, P. R., Love, P. E. D., and Baccarini, D. (2009). Bills of Quantities: nemesis or nirvana? Structural Survey 27, 99-108.

Dean, R. P., and McClendon, S. (2007). Specifying and Cost Estimating with BIM.

Dorst, K., and Dijkhuis, J. (1995). Comparing paradigms for describing design activity. Design Studies 16, 261-274.

Ericsson, K. A., and Simon, H. A. (1993). "Protocol analysis : verbal reports as data," MIT Press, Mass.

Flager, F., Welle, B., Bansal, P., Soremekun, G., and Haymaker, J. (2009). Multidisciplinary Process Integration \& Design Optimization of a Classroom Building. 14, 595-612.

Gero, J., and Tang, H.-H. (2001). The differences between retrospective and concurrent protocols in revealing the process-oriented aspects of the design process. Design Studies 22, 283-295.

Gero, J. S. (1990). Design prototypes: a knowledge representation schema for design. AI Magazine 11, 26-36.

Gero, J. S., and Kannengiesser, U. (2004). The situated function-behaviour-structure framework. Design studies 25, 373-391.

Gero, J. S., and Mc Neill, T. (1998). An approach to the analysis of design protocols. Design Studies 19, 21-61.

Gujarathi, G. P., and Ma, Y. S. (2011). Parametric CAD/CAE integration using a common data model. Journal of Manufacturing Systems 30, 118-132. 
Iordanova, I., Tidafi, T., Guité, M., De Paoli, G., and Lachapelle, J. (2009). Parametric methods of exploration and creativity during architectural design: A Case study in the design studio. In "CAADFutures2009", pp. 423-439.

Jadid, M. N., and Idrees, M. M. (2007). Cost estimation of structural skeleton using an interactive automation algorithm: A conceptual approach. Automation in Construction 16, 797-805.

Kan, J. W. T., and Gero, J. S. (2008). Acquiring information from linkography in protocol studies of designing. Design Studies 29, 315-337.

Kolarevic, B. (2003). "Architecture in the digital age : design and manufacturing," Spon Press, New York, NY.

Kuusela, H., and Pallab, P. (2000). A comparison of concurrent and retrospective verbal protocol analysis. American Journal of Psychology 113, 387-404.

Kwon, O., Lee, G., Kim, S., Sin, Y., Hwang, J., and Chae, K. (2009). Interoperability Issues in Cross-disciplinary Collaborations of Irregularly Shaped Building: the Case of Dongdaemun Design Plaza and Park In "The 3rd International Conference on Construction Engineering and Management and The 6th International Conference on Construction Project Management (ICCEM ICCPM 2009)", pp. 331-337. Nanyang Technological University of Singapore, Jeju, Korea.

Lee, J. H., Gu, N., Jupp, J., and Sherratt, S. (2012). Evaluating Creativity in Parametric Design Processes and Products: A Pilot Study. In "DCC2012" (J. Gero, ed.). Springer, Texas.

Lowe, D., and Skitmore, M. (1994). Experiential learning in cost estimating. Construction Management and Economics 12, 423-431.

Lowe, D., and Skitmore, M. (2007). Organisational learning style and practitioner competence. Journal of Financial Management of Property and Construction 11.

Maher, M. L. (2008). Keynote: Creativity and Computing in construction. In "Annual Conference of the Australian and New Zealand Architectural Science Association (ANZAScA 08)". University of Newcastle, Australia

Ogunlana, S. O. (1989). Accuracy in Design Cost Estimating, University of Technology, Loughborough, UK.

Olatunji, O. A. (2011). Modelling the costs of corporate implementation of building information modelling. Journal of Financial Management of Property and Construction 16, 211-231.

Olatunji, O. A. (2012). The impact of building information modelling on estimating practice: analysis of perspectives from four organizational business models. Doctor of Philosophy Dissertation, University of Newcastle, Newcastle, Australia.

Olatunji, O. A. (2014). Synthesis of views on BIM, procurement and contract management. ICE Proceedings: Management, Procurement and Law Accepted.

Pinto, J. K., and Mantel, S. J., Jr. (1990). The causes of project failure. Engineering Management, IEEE Transactions on 37, 269-276.

Schnabel, M. A. (2007). Parametric Designing in Architecture. In "CaadFutures2007" (A. Dong, V. Moere and J. S. Gero, eds.), pp. 237-250. Springer, Sydney.

Skitmore, M., and Wilcock, J. (1994). Estimating processes of smaller builders. Construction Management and Economics 12, 139 - 154.

Skitmore, R. M., and Patchell, B. R. T. (1990). Development in contract price forecasting and bidding techniques. In "Quantity Surveying Techniques: New 
Directions" (P. S. Brandon, ed.), pp. 75-120. Blackwell Scientific.

Sutrisna, M., Buckley, K., Potts, K., and Proverbs, D. (2005). "A decision support tool for the valuation of variations on civil engineering projects ". Royal Institution of Chattered Surveyors (RICS), London.

Whyte, J., Bouchlaghem, N., Thorpe, A., and McCaffer, R. (2000). From CAD to virtual reality: modelling approaches, data exchange and interactive 3D building design tools. Automation in Construction 10, 43-55.

Woodbury, R. (2010). "Elements of Parametric Design," Taylor and Francis.

Yang, J., and Han, S. (2006). Repairing CAD model errors based on the design history. Computer-Aided Design 38, 627-640.

Zyskowski, P. (2009). The world according to BIM: Parts 1 - 3. Cadalyst.com/aec. 